\title{
A COMBINATORIAL MODEL FOR SERIES-PARALLEL NETWORKS
}

\author{
BY \\ THOMAS BRYLAWSKI
}

\begin{abstract}
The category of pregeometries with basepoint is defined and explored. In this category two important operations are extensively characterized: the series connection $S(G, H)$, and the parallel connection $P(G, H)=\tilde{S}(\tilde{G}, \tilde{H})$; and the latter is shown to be the categorical direct sum. For graphical pregeometries, these notions coincide with the classical definitions.

A pregeometry $F$ is a nontrivial series (or parallel) connection relative to a basepoint $p$ iff the deletion $F \mid p$ (contraction $F / p$ ) is separable. Thus both connections are $n$-ary symmetric operators with identities and generate a free algebra. Elements of the subalgebra $A\left[C_{2}\right]$ generated by the two point circuit are defined as series-parallel networks, and this subalgebra is shown to be closed under arbitrary minors. Nonpointed series-parallel networks are characterized by a number of equivalent conditions:

1. They are in $A\left[C_{2}\right]$ relative to some point.

2. They are in $A\left[C_{2}\right]$ relative to any point.

For any connected minor $K$ of three or more points:

3. $K$ is not the four point line or the lattice of partitions of a four element set.

4. $K$ or $\tilde{K}$ is not a geometry.

5 . For any point $e$ in $K, K \mid e$ or $K / e$ is separable.

Series-parallel networks can also be characterized in a universally constructed ring of pregeometries generalized from previous work of W. Tutte and A. Grothendieck. In this Tutte-Grothendieck ring they are the pregeometries for which the Crapo invariant equals one. Several geometric invariants are directly calculated in this ring including the complexity and the chromatic polynomial. The latter gives algebraic proofs of the two and three color theorems.
\end{abstract}

1. Introduction. The notion of series-parallel networks goes back to MacMahon [10], who studied their enumeration. After a long period of neglect, it was revived in a well-known paper by Shannon [14], and since then a flurry of papers has appeared exploring their structure and applying them in various directions. A particularly significant work is the paper by Duffin [9], where most of the known results on series-parallel networks are developed.

In our paper we initiate (and to some extent complete) the study of series-parallel networks by completely different techniques. Our starting point is the theory of

Received by the editors November 24, 1969.

AMS 1969 subject classifications. Primary 0540, 0555, 9430, 5510, 5060, 0527; Secondary $1810,1393,0545$.

Key words and phrases. Matroid, pointed pregeometry, basepoint, isthmus, loop, contraction, deletion, series connection, parallel connection, pointed direct sum, pushout, complexity, Möbius function, Crapo invariant, chromatic polynomial, graph coloring, Hadwiger conjecture, two color theorem, Tutte polynomial, Tutte-Grothendieck ring, series-parallel network, essentially series, essentially parallel, series-parallel irreducible. 
combinatorial pregeometries (nées matroids) begun by Whitney [19] and recently expounded by Crapo and Rota [7]. (See \$2 where the basic concepts are summarized.) We introduce the concept of a combinatorial pregeometry with a distinguished basepoint, an idea suggested to the author by Gian-Carlo Rota. In the first few sections we develop some of the general theory of basepointed pregeometries, which appear to have many further applications.

A pregeometry is graphical when its point-circuit incidence relation is the same as the edge-circuit incidence relation of some graph. In that case the basepoint corresponds to a distinguished edge in the graph and hence to two distinguished vertices (or "ports" in the literature of series-parallel networks). Circuits containing this basepoint will therefore be in 1-1 correspondence with paths joining the two ports in the classical network.

We find this an appropriate model for a number of reasons: the geometry of the network demonstrates the essential duality between the series and the parallel connection-the fact that the (Whitney) dual of a series connection of networks is the parallel connection of their duals. In addition, for electrical and historical reasons one wants two networks isomorphic under permutation of elements in series as well as the usual graph-theoretic isomorphism. But these are exactly the conditions under which the geometries of the two networks are isomorphic.

Except for Shannon's notion of "confluence", all classical characterizations of series-parallel networks are derived by purely combinatorial methods, as well as two important others-one in terms of separability (one-connectedness in graph theory) and the other in terms of an invariant of a ring associated with all pointed pregeometries. This ring is a generalization of a ring introduced for graphs more than twenty years ago by W. T. Tutte [15], and its construction is reminiscent of constructions recently used with great success in the field of algebraic geometry by A. Grothendieck. For this reason we have decided to call it the Tutte-Grothendieck ring.

The remarkable fact about the Tutte-Grothendieck ring is that purely combinatorial properties of series-parallel networks can be translated by a systematic process into algebraic properties of the ring. Specifically, to every series-parallel network we associate a polynomial in four variables in the ring (which we call the Tutte polynomial, after previous work of Henry Crapo [5]). By evaluating the variables of this polynomial at appropriate integers, we obtain the values of various important combinatorial invariants of the network, such as the number of forests and number of spanning trees.

One of the more remarkable results of the present theory is that the classical problem of coloring of graphs can be completely translated into simple algebraic properties of the Tutte-Grothendieck ring. In particular, we are able to obtain the solution of the coloring problem for series-parallel networks by purely algebraic techniques. Admittedly, this coloring problem is not very difficult (it is not hard to prove by standard methods that every such graph can be colored in at most three 
colors); nevertheless, the introduction of purely algebraic techniques for proving the same results, such as when two instead of three colors are sufficient, seems promising for future work on the coloring problem. In fact we present what amounts to algebraic proofs for $k=2$ and 3 of the famous Hadwiger conjecturethat a graph with no subgraph homeomorphic to the complete $k$-graph can be colored in $k-1$ colors--while the case $k=4$ implies the four-color theorem for planar graphs.

The idea that a Tutte-Grothendieck ring (and the more general concept, the Tutte-Grothendieck group) could be defined for all combinatorial pregeometries was first introduced by G.-C. Rota in his Hedrick Lectures [13]. A systematic development of these ideas appears in the author's thesis.

The author is indebted to Professors Henry Crapo, Robert Norman, and GianCarlo Rota for several conversations on this subject.

\section{Basic definitions.}

2.1. This section surveys the relevant notions of the underlying category for our work, $\boldsymbol{G}$, the category of finite combinatorial pregeometries and strong maps discussed in Crapo and Rota [7]. The reader is advised to read these basic definitions rapidly and refer to them as they come up in the paper.

A finite pregeometry or matroid, $G$, is a finite set, denoted $|G|$, of points with a closure relation satisfying the exchange property: For any points $p, q \in G$ and any subset $P \subseteq|G|$, if $p \in \mathrm{Cl}(P \cup\{q\})$ but $p \notin P$, then $q \in \mathrm{Cl}(P \cup\{p\})$. A geometry is a pregeometry in which the empty set and each point are all closed. The lattice, $L$, of closed sets or flats of a pregeometry is called a geometric lattice and is characterized as a finite, semimodular, point lattice. In such lattices, each lattice element $x$ is the supremum of atoms representing closures of points and each has a well-defined rank, $r(x)$, equal to the length of any maximal chain from the 0 element (representing the closure of the empty set) to $x$. The semimodular law for $L$ states that for all $x, y \in L, r(x)+r(y) \geqq r(x \wedge y)+r(x \vee y) . r(A)$, the rank of a set of points $A \subseteq|G|$ is defined as $r(\bar{A})$ in the associated geometric lattice. Hence, $r(G)$, the rank of the pregeometry is $r(1)$ in the lattice. A set of points $A \subseteq|G|$ represents a spanning set for $G$ if $\bar{A}=G$. The cardinality of a pregeometry $G$ (or point set $A \subseteq|G|$ ) will be denoted $\|G\|$ (or $\|A\|$ ) and a set of points, $A$, is independent if $r(A)=\|A\|$. Otherwise, $r(A)<\|A\|$ and $A$ is dependent. An independent spanning set is called a basis.

A strong map from a pregeometry $G$ into $H$ is a function $f:|G| \cup\{0\} \rightarrow|H| \cup\{0\}$ (where " 0 " stands for the empty set in $G$ and $H$ respectively) such that $f(0)=0$ and the inverse image of any closed set in $H$ is closed in $G$. Pregeometries $G$ and $H$ are isomorphic denoted $G \simeq H$ if there is a 1-1 correspondence, $f$, between the points of $G$ and $H$ and the closed sets of $G$ and $H$ such that for any point $p$ and closed set $K$, $p \in K$ iff $f(p) \in f(K)$. An isomorphism class of pregeometries denoted [G] is the class of all pregeometries isomorphic to $G$.

A pregeometry on the point set $|G|$ can be uniquely determined by $C(G)$, the family of minimal dependent sets or circuits of $|G|$. A family $\boldsymbol{F}$ of subsets is the 
circuit set for some pregeometry if no subset in $\boldsymbol{F}$ properly contains another and the subsets satisfy the circuit elimination property $C^{*}$ : If $C_{1}$ and $C_{2}$ are two distinct elements of $\boldsymbol{F}$ and $e \in C_{1} \cap C_{2}$ then the set difference $\left(C_{1} \cup C_{2}\right) \mid\{e\}$ is dependent and contains an element $C_{3} \in \boldsymbol{F}$. A pregeometry is an $n$-point circuit if $\|G\|=n$ and $C(G)=\{|G|\} . C \in C(G)$ is an even circuit if $\|C\|$ is even. A subset $A \subseteq|G|$ contains a broken circuit if for some $C \in C(G)$ and some $p \in C, C \mid\{p\} \subseteq A$ but $C \nsubseteq A$.

$G$ may also be uniquely determined from its set of bases, $B(G)$. A family $\boldsymbol{F}$ of incomparable subsets is the set of bases for some pregeometry if $\boldsymbol{F}$ satisfies the basis exchange axiom $\boldsymbol{B}^{*}$ : For all $B_{1}$ and $B_{2}$ in $\boldsymbol{F}$ and $p \in B_{1}$ there exists $q \in B_{2}$ such that $\left(B_{1} \mid\{p\}\right) \cup\{q\}$ is also in $\boldsymbol{F}$.

The (Whitney) dual of $G, \widetilde{G}$, is the unique pregeometry on the point set $|G|$ with a set of bases consisting of base complements of $G$. Hence $B \in \boldsymbol{B}(\widetilde{G})$ iff $|G| \mid B \in \boldsymbol{B}(G)$. A pregeometry is self-dual if $G \simeq \widetilde{G}$.

$G$ is the direct sum of two pregeometries: $G_{1} \oplus G_{2}$ if the points of $G,|G|$, and circuits of $G, C(G)$, are the disjoint unions $\left|G_{1}\right| \cup\left|G_{2}\right|$ and $C\left(G_{1}\right) \cup C\left(G_{2}\right)$ respectively. $G_{1}$ is then said to be a direct sum factor of $G$, and $G$ is said to be separable. If no such nontrivial direct sum decomposition exists, any two distinct points of $G$ are contained in a circuit and $G$ is termed connected. A one point direct sum factor, $p$, is an isthmus if it is in no circuits of $G$ and a loop if it is itself a circuit.

If $p \in|G|$ we define two derived pregeometries on the point set $|G| \mid\{p\}$ : the deletion, $G \backslash p$; and the contraction, $G / p$. If $A \subseteq|G| \mid\{p\}$, and $\bar{A}$ denotes its closure in $G$ : then the closure of $A$ in $G \backslash p$ is defined as $\bar{A} \backslash\{p\}$ while its closure in $G / p$ is defined as $\operatorname{Cl}(A \cup\{p\}) \mid\{p\}$. If $D \subseteq|G|$, the subgeometry $G \backslash D$ is defined as a sequence of deletions by points in $D$. Similarly we define the contraction $G / D$ as a sequence of contractions. An arbitrary sequence of contractions and deletions is called a minor.

An invariant is a function $f$ defined on the class of all pregeometries such that $f(G)=f(H)$ if $G \simeq H$. Examples of invariants used in this paper include $c(G)$, the complexity or number of bases of $G ; I(G)$, the number of independent sets; and $\mu(G)$, the Möbius function which is defined as $\mu(0,1)$ evaluated on the geometric lattice $L$ associated with $G$, where for $x \leqq y, \mu(x, y)$ is given by the recursion $\mu(x, x)=1, \mu(x, y)=-\sum_{x \leqq z<y} \mu(x, z)$. Two other invariants evaluated on $L$ are the chromatic polynomial, $\chi(G)=\sum_{x \in L} \mu(0, x) \lambda^{r(1)-r(x)}$ and $\beta(G)$, the Crapo invariant which is explored in [3] with distinguishing properties; $\beta(G)=\beta(G \mid e)+\beta(G / e)$ if $e \in G$ is neither an isthmus nor a loop; $\beta(G) \geqq 0 ; \beta(G)=0$ iff $G$ is separable; and $\beta(G)=\beta(\tilde{G})$ for all $\|G\|>1$.

2.2. Let $C$ denote the category of pointed pregeometries; that is, ordered pairs $(G, p)$ where $G$ is a pregeometry on the point set $|G|$ and $p \in|G|$. Morphisms in this category are strong maps which preserve basepoint, so $f \in \operatorname{Hom}\left(\left(G, p_{G}\right),\left(H, p_{H}\right)\right)$ if $f$ is a strong map and $f\left(p_{G}\right)=p_{H}$.

\section{Conventions.}

3.1. All unions $A \cup B$ will be assumed to be disjoint unless it is clear that $A$ and $B$ are both subsets of the same set. 
3.2. $A \backslash B$ denotes set difference: elements in $A$ but not in $B$.

3.3. If $\boldsymbol{A}$ and $\boldsymbol{B}$ are two families of subsets of $A$ and $B$ respectively, then $\boldsymbol{A} \times \boldsymbol{B}$ will represent all subsets $C \cup D$ of $A \cup B$ where $C \in A$ and $D \in B$.

3.4. When no confusion will arise we will denote the pair $\left(G, p_{G}\right)$ by $(G, p)$, and sometimes $G$.

3.5. Given a pointed pregeometry $(G, p)$ and a family $\boldsymbol{F}$ of subsets of $G$, we define $\boldsymbol{F}^{\prime}(G)$ to be the subfamily of $\boldsymbol{F}$ whose members do not contain the basepoint $p$ and $\boldsymbol{F}^{\prime \prime}(G)$ to be the family of all those subsets $H$ of $|G| \mid\{p\}$ such that $H \cup\{p\} \in \boldsymbol{F}$. Hence $\boldsymbol{F}=\boldsymbol{F}^{\prime} \cup\left(\boldsymbol{F}^{\prime \prime} \times\{p\}\right)$. In particular $|G|^{\prime}=|G| \mid\{p\}$.

4. The series connection. There are two important operations on pointed pregeometries: the series connection and the parallel connection. We now define and explore the series connection.

Definition 4.1. Given two pregeometries $\left(G, p_{G}\right)$ and $\left(H, p_{H}\right)$ the series connection $(F, p)=S\left(\left(G, p_{G}\right),\left(H, p_{H}\right)\right)$ is the pregeometry defined on the point set $|F|$ $=|F|^{\prime} \cup\{p\}$, where $|F|^{\prime}=|G|^{\prime} \cup|H|^{\prime}$ and whose circuits $C((F, p))$ are given by the two families:

$$
C^{\prime}(F)=C^{\prime}(G) \cup C^{\prime}(H), \quad C^{\prime \prime}(F)=C^{\prime \prime}(G) \times C^{\prime \prime}(H) .
$$

Proposition 4.2. The family $C(F)$ defined by (4.1) above is the set of circuits for some pregeometry.

Proof. Clearly no circuit denoted above is a proper subset of another. We must show that $C(F)$ satisfies the circuit elimination axiom $C^{*}$. Assume $C_{1}$ and $C_{2}$ are two unequal circuits in $C(F)$ and $e \in C_{1} \cap C_{2}$. By symmetry we may assume $e \in G$ (possibly $e$ is the basepoint $p$ ).

If $C_{1}$ and $C_{2}$ are both in the family $C^{\prime}(F)$ and if they have nontrivial intersection, then they must both lie in the subfamily $C^{\prime}(G)$, in which case $C^{*}$ follows from $C^{*}$ in $G$.

If $C_{1} \in C^{\prime}(G)$ and $C_{2} \in C^{\prime \prime}(F) \times\{p\}$, then $C_{1}$ and $\left(C_{2} \cap|G|^{\prime}\right) \cup\left\{p_{G}\right\}$ are both circuits of $G$ and $C^{*}$ applied in $G$ gives a circuit of $G, C_{3}^{\prime}$. If $C_{3}^{\prime}$ is in the subfamily $C^{\prime}(G) \subseteq C(F)$ we are done. Otherwise $C_{3}^{\prime} \in C^{\prime \prime}(G) \times\left\{p_{G}\right\}$ and $C_{3}=C_{3}^{\prime} \cup\left(C_{2} \cap|H|^{\prime}\right)$ $\cup\{p\}$ gives the desired element of $C^{\prime \prime}(F) \times\{p\}$.

If the subsets $C_{1}$ and $C_{2}$ are both in the family $C^{\prime \prime}(F) \times\{p\}$, and if $C_{1} \cap|G| \neq C_{2}$ $\cap|G|$ we may proceed as in the previous case to find a circuit $C_{3}^{\prime}$ in $G$ and we form the circuit $C_{3}$ of $F$ adding $\left(C_{2} \cap|H|^{\prime}\right) \cup\{p\}$ if necessary. If $C_{1} \cap|G|=C_{2} \cap|G|$, then necessarily $C_{1} \cap|H|^{\prime} \neq C_{2} \cap|H|^{\prime}$ and applying $C^{*}$ in $H$ to these above two circuits which both contain the basepoint $p_{H}$ we obtain a circuit $C_{3} \in C^{\prime}(H)$ which of course does not contain the point $e \in G$.

Proposition 4.3. $S(G, H)=S(H, G)$ and $S(G, S(H, I))=S(S(G, H), I)$ so we can view $S$ as an n-ary symmetric operator. Further, if $(H, p)$ is a loop then $S(G, H)=G$.

Proof. These remarks follow trivially from (4.1). 
Proposition 4.4. If $p$ is not an isthmus in $G$ or $H$, the bases $B(F)$ of $S(G, H)$ are given by the following families:

$$
\begin{aligned}
& \boldsymbol{B}^{\prime}(F)=\boldsymbol{B}^{\prime}(G) \times \boldsymbol{B}^{\prime}(H), \\
& \boldsymbol{B}^{\prime \prime}(F)=\left(\boldsymbol{B}^{\prime \prime}(G) \times \boldsymbol{B}^{\prime}(H)\right) \cup\left(\boldsymbol{B}^{\prime}(G) \times \boldsymbol{B}^{\prime \prime}(H)\right) .
\end{aligned}
$$

Proof. The bases of $F$ are exactly those maximal subsets of points which contain no circuit. Clearly none of the above bases contains any circuit in $C^{\prime}(F)$. If the subset $B$ in $B^{\prime \prime}(G) \times \boldsymbol{B}^{\prime}(H)$ contained the circuit $C$ where $C \in C^{\prime \prime}(F) \times\{p\}$ then $C \cap|G| \subseteq(B \cap|G|) \cup\{p\}$, a basis for $G$. But $C \cap|G|$ is a circuit of $G$-a contradiction.

Now assume $I$ is an independent subset of $F$. If $p \notin I$, then the subset $I \cap|G|$ is independent in $G \backslash\{p\}$ and is contained in the basis for $G, B_{G} \in \boldsymbol{B}^{\prime}(G)$ while $I \cap|H| \subseteq B_{H} \in \boldsymbol{B}^{\prime}(H)$. Hence, the subset $I$ is contained in a basis $B \in \boldsymbol{B}^{\prime}(G) \times \boldsymbol{B}^{\prime}(H)$.

If the basepoint $p$ is in $I$, let $I^{\prime}=I \backslash\{p\}$. Then the subset $I^{\prime}$ is independent and as above: $I^{\prime} \cap|G| \subseteq B_{G} \in B^{\prime}(G)$ and $I^{\prime} \cap|H| \subseteq B_{H} \in B^{\prime}(H)$. Assume $I^{\prime} \cap|G|$ is contained in no subset $B_{G} \in \boldsymbol{B}^{\prime \prime}(G)$. Then $\left(I^{\prime} \cap|G|\right) \cup\{p\}$ contains a circuit $C_{G}$ where $C_{G} \in C^{\prime \prime}(G) \times\{p\}$. Similarly, if $I^{\prime} \cap H \nsubseteq B_{H}$ for all $B_{H} \in \boldsymbol{B}^{\prime \prime}(H)$, then $\left(I^{\prime} \cap H\right) \cup\{p\}$ contains a circuit $C_{H}$ containing $p$ and the independent set $I \supseteq\left(C_{G} \cup C_{H}\right) \in C^{\prime \prime}(F) \times\{p\}$-a contradiction; so $I^{\prime} \cap H \subseteq B_{H} \in B^{\prime \prime}(H)$. Hence the independent set $I$ is contained in a basis $B \in\left(\boldsymbol{B}^{\prime}(G) \times \boldsymbol{B}^{\prime \prime}(H)\right) \times\{p\}$.

Proposition 4.5. If the basepoint $p$ is an isthmus of the pregeometry $G$, then the family $C^{\prime \prime}(G)$ is empty and $C(F)=C^{\prime}(G) \cup C^{\prime}(H)$. Hence $F \simeq\{p\} \oplus(G \backslash p)$ $\oplus(H \backslash p)$ and $\boldsymbol{B}^{\prime}(F)$ is empty; while $\boldsymbol{B}^{\prime \prime}(F)=\boldsymbol{B}^{\prime \prime}(G) \times \boldsymbol{B}^{\prime \prime}(H)$ if $p$ is an isthmus of $H$ and $\boldsymbol{B}^{\prime \prime}(F)=\boldsymbol{B}^{\prime \prime}(G) \times \boldsymbol{B}^{\prime}(H)$ otherwise. If $p$ is neither a loop nor an isthmus, then $\boldsymbol{B}^{\prime}(F)$ are the bases for the deletion $F \backslash p$ while $\boldsymbol{B}^{\prime \prime}(F)$ form the bases for the contraction $F / p$. In any case, $C^{\prime}(F)=C(F \mid p)$.

Proof. From (4.1) and (4.4).

Proposition 4.6. If the pointed pregeometries $G$ and $H$ each have at least two points, then $F=S(G, H)$ is connected if and only if both $G$ and $H$ are connected.

Proof. If $G$ and $H$ are both connected and the points $e$ and $e^{\prime}$ are both in the subset $|G|^{\prime}$, then there is a circuit $C \in C^{\prime}(G)$ containing both. But then $C \in C^{\prime}(F)$. If $e \in G, e^{\prime} \in H$ (either could be the basepoint $p$ ); then since neither $G$ nor $H$ is $\{p\}$ but both are connected, $e$ is in a circuit with $p$ in $G$ and $e^{\prime}$ is in a circuit with $p$ in $H$. So $e$ and $e^{\prime}$ are in a circuit containing $p$ in $F$ since $C^{\prime \prime}(F)=C^{\prime \prime}(G) \times C^{\prime \prime}(H)$.

Conversely, if $G$ is not connected, $\exists e, e^{\prime} \in G$ (possibly $e^{\prime}$ is the basepoint $p$ ) such that no circuit of $G$ contains both. Surely no circuit in the family $C(H)$ contains $e$ and hence no circuit in the family $C(F)$ contains both.

Proposition 4.7. If the point $e \in|G|^{\prime}$ and $F=S(G, H)$ then $F / e=S(G / e, H)$ and $F \mid e=S(G \backslash e, H)$. Hence the series operation commutes with contraction and deletion. 
Proof. Since $C^{\prime}(F)$ contains $C^{\prime}(G), e$ is a loop of $G$ iff it is a loop of $F$. If $e$ is not a loop, the bases for $G / e(F / e)$ are those subsets $B$ of $|G|^{\prime}\left(|F|^{\prime}\right)$ such that $B \cup\{e\}$ is a basis of $G(F)$. If $e$ is a loop, the bases for $G / e(F / e)$ and $G(F)$ are the same. In any case, if $p$ is never an isthmus,

$$
\begin{aligned}
B^{\prime}(F / e) & =B^{\prime}(G / e) \times B^{\prime}(H), \\
B^{\prime \prime}(F / e) & =\left(B^{\prime \prime}(G / e) \times B^{\prime}(H)\right) \cup\left(B^{\prime}(G / e) \times B^{\prime \prime}(H)\right) .
\end{aligned}
$$

The case where $e$ is deleted is proved analogously, as is the case where $p$ is an isthmus.

Corollary 4.8. If $F=S(G, H)$ and the basepoint $p$ is not an isthmus of $G$, then $(F, p) /|G|^{\prime}=(H, p)$.

Proof. Since $p$ is not an isthmus, $G /|G|^{\prime}$ is a loop and the result follows from (4.7) and (4.3).

Proposition 4.9. If $(F, p)=S((G, p),(H, p))$ then the deletion $F \mid p$ is equal to the direct sum $(G \mid p) \oplus(H \mid p)$.

Proof. Both pregeometries are defined on the same point set $|F|^{\prime}=|G|^{\prime} \cup|H|^{\prime}$ and since $F=G_{1} \oplus G_{2}$ iff they are defined on the same point set and $C(F)=C\left(G_{1}\right)$ $\cup C\left(G_{2}\right)$ (disjoint); the proposition follows from (4.1) and (4.5).

This proposition has the following converse:

Proposition 4.10. If $F$ is a connected pregeometry and the deletion $F \mid p$ is the direct sum of two pregeometries, $G^{\prime} \oplus H^{\prime}$, then $F$ is isomorphic to the series connection $S\left(F /\left|G^{\prime}\right|, F /\left|H^{\prime}\right|\right)$.

Proof. We define the pregeometries $(G, p)$ and $(H, p)$ on the point sets $\left|G^{\prime}\right| \cup\{p\}$ and $\left|H^{\prime}\right| \cup\{p\}$ respectively by defining the families $\boldsymbol{B}^{\prime}(G)=\boldsymbol{B}\left(G^{\prime}\right) ; \boldsymbol{B}^{\prime}(H)=\boldsymbol{B}\left(H^{\prime}\right)$; $\boldsymbol{B}^{\prime \prime}(G)=\left\{g \subseteq\left|G^{\prime}\right| \mid g \cup h \in \boldsymbol{B}^{\prime \prime}(F)\right.$ for some $\left.h \in \boldsymbol{B}^{\prime}(H)\right\} ;$ and $\boldsymbol{B}^{\prime \prime}(H)=\left\{h \subseteq\left|H^{\prime}\right| \mid g\right.$ $\cup h \in \boldsymbol{B}^{\prime \prime}(F)$ for some $\left.g \in \boldsymbol{B}^{\prime}(G)\right\}$. Then by hypothesis $\boldsymbol{B}^{\prime}(F)=\boldsymbol{B}^{\prime}(G) \times \boldsymbol{B}^{\prime}(H)$. We need to show that $\boldsymbol{B}^{\prime \prime}(F)=\left(\boldsymbol{B}^{\prime}(G) \times \boldsymbol{B}^{\prime \prime}(H)\right) \cup\left(\boldsymbol{B}^{\prime \prime}(G) \times \boldsymbol{B}^{\prime}(H)\right)$. Assume for example $g \cup h \in \boldsymbol{B}^{\prime}(G) \times \boldsymbol{B}^{\prime \prime}(H)$. Then by definition of $\boldsymbol{B}^{\prime \prime}(H), g^{\prime} \cup h \in \boldsymbol{B}^{\prime \prime}(F)$ for some subset $g^{\prime}$ in $\boldsymbol{B}^{\prime}(G)$. So $g^{\prime} \cup h \cup\{p\}$ is a basis for $F$ and $h$ is an independent subset of $H^{\prime}$. Hence $g \cup h$ is an independent subset of the subgeometry $G^{\prime} \oplus H^{\prime}$ and therefore an independent subset of $F$ of cardinality one less than a basis. By basis exchange in $F$ we can add a point from the subset $g^{\prime} \cup h \cup\{p\}$ to $g \cup h$ and get a basis for $F$. But no point in $G^{\prime}$ can be added as $g$ is already a basis for $G^{\prime}$. The only other point in the set difference is $p$; hence, $g \cup h \in \boldsymbol{B}^{\prime \prime}(F)$. The family $\boldsymbol{B}((G, p))$ $=\boldsymbol{B}^{\prime}(G) \cup\left(\boldsymbol{B}^{\prime \prime}(G) \times\{p\}\right)$ satisfies the basis exchange axiom since $F$ does and since any two bases in $\boldsymbol{B}(G)$ can be extended by the same subset $h$ in $\boldsymbol{B}^{\prime}(H)$ to give two bases in $F$. Similarly, $(H, p)$ is a pointed pregeometry; and $F=S(G, H)$. By (4.8), $G=F /|H|^{\prime}=F /\left|H^{\prime}\right|$ and $H=F /\left|G^{\prime}\right|$. 
Corollary 4.11. A connected pregeometry $(F, p)$ of more than one point has a unique series decomposition into series irreducible connected nontrivial pregeometries $\left(G_{1}, p\right), \ldots,\left(G_{n}, p\right)$ such that $F=S\left(G_{1}, \ldots, G_{n}\right)$, which is unique up to permutation of the $G_{i}$ 's.

Proof. This follows from the existence and uniqueness of direct sum decomposition and (4.10), (4.9), and (4.3).

Proposition 4.12. The closed sets $K(F)$ of $F=S(G, H)$ are given by the following families:

$$
\begin{aligned}
& \boldsymbol{K}^{\prime}(F)=\left(\boldsymbol{K}^{\prime}(G) \times \boldsymbol{K}^{\prime}(H)\right) \cup\left(\boldsymbol{K}^{\prime}(G) \times \boldsymbol{K}^{\prime \prime}(H)\right) \cup\left(\boldsymbol{K}^{\prime \prime}(G) \times \boldsymbol{K}^{\prime}(H)\right), \\
& \boldsymbol{K}^{\prime \prime}(F)=\left(\boldsymbol{K}^{\prime}(G) \times \boldsymbol{K}^{\prime}(H)\right) \cup\left(\boldsymbol{K}^{\prime \prime}(G) \times \boldsymbol{K}^{\prime \prime}(H)\right) .
\end{aligned}
$$

Proof. A set of points $A \subseteq|F|$ is closed iff it contains no broken circuit, i.e., $\forall C \in C(F),\|C \backslash A\| \neq 1$. If a set is not closed the circuit $C$ can be chosen to contain any given point $q \in \bar{A} \backslash A$. Since $C^{\prime}(G)=C\left(G^{\prime}\right)$ and $C^{\prime}(H)=C\left(H^{\prime}\right)$ and since these circuits do not contain the basepoint $p$ and hence $|C| A|=| C|(A \cup\{p\})|$, it follows that a set $A$ contains no broken circuits from the family of circuits $C^{\prime}(F)$ iff $A \cap\left|G^{\prime}\right| \in K^{\prime}(G) \cup K^{\prime \prime}(G)$ and $A \cap\left|H^{\prime}\right| \in K^{\prime}(H) \cup K^{\prime \prime}(H)$. We need only consider those sets $A$ such that $A \backslash\{p\} \in\left(K^{\prime}(G) \cup K^{\prime \prime}(G)\right) \times\left(K^{\prime}(H) \cup K^{\prime \prime}(H)\right)$ and which do not contain a broken circuit from any circuit $C \in C^{\prime \prime}(F) \times\{p\}$.

For a circuit $C$ in $C^{\prime \prime}(G) \times C^{\prime \prime}(H) \times\{p\}$ and set $A$ in $|F|^{\prime}$, the basepoint $p$ is in the set difference $C \backslash A$. Another point will also be in the difference unless the subset $A \cap\left|G^{\prime}\right| \notin K^{\prime}(G)$ and $A \cap\left|H^{\prime}\right| \notin K^{\prime}(G)$. This characterizes the closed sets in $K^{\prime}(F)$.

For a set $A$ containing $p, p \notin(C \backslash A)$ so the set will contain a broken circuit from $C^{\prime \prime}(F) \times\{p\}$ iff $\left\|\left(C \cap\left|G^{\prime}\right|\right) \mid\left(A \cap\left|G^{\prime}\right|\right)\right\|=1$ or $\left\|\left(C \cap\left|H^{\prime}\right|\right) \mid\left(A \cap\left|H^{\prime}\right|\right)\right\|=1$ but not both. This holds iff $A \backslash\{p\}$ is in either of the families $\left(\boldsymbol{K}^{\prime \prime}(G) \backslash \boldsymbol{K}^{\prime}(G)\right) \times\left(\boldsymbol{K}^{\prime}(H) \backslash \boldsymbol{K}^{\prime \prime}(H)\right)$ or $\left(\boldsymbol{K}^{\prime}(G) \backslash \boldsymbol{K}^{\prime \prime}(G)\right) \times\left(\boldsymbol{K}^{\prime \prime}(H) \backslash \boldsymbol{K}^{\prime}(H)\right)$.

5. The parallel connection. We now explore an operation dual to the series connection-the parallel connection.

Definition 5.1. For two pregeometries $\left(G, p_{G}\right)$ and $\left(H, p_{H}\right)$ we define the parallel connection

$$
(F, p)=P\left(\left(G, p_{G}\right),\left(H, p_{H}\right)\right)=\tilde{S}(\tilde{G}, \tilde{H}) ;
$$

where $(\tilde{K}, p)$ is the (unique) pointed pregeometry dual to $(K, p)$ with the same basepoint.

Note since $(\tilde{K})^{\sim}=K, \widetilde{P}(\tilde{G}, \tilde{H})=S(G, H)$.

Proposition 5.2. If $p$ is not a loop in $G$ or $H$, the bases of the parallel connection $F=P(G, H)$ are given by the following families:

$$
\begin{aligned}
& \boldsymbol{B}^{\prime}(F)=\left(\boldsymbol{B}^{\prime}(G) \times \boldsymbol{B}^{\prime \prime}(H)\right) \cup\left(\boldsymbol{B}^{\prime \prime}(G) \times \boldsymbol{B}^{\prime}(H)\right), \\
& \boldsymbol{B}^{\prime \prime}(F)=\boldsymbol{B}^{\prime \prime}(G) \times \boldsymbol{B}^{\prime \prime}(H) .
\end{aligned}
$$


Proof. Since the set $B$ is a basis of $\tilde{G}$ iff its complement $|G| \mid B$ is a basis of $G$, and since the basepoint $p$ is in $B$ iff $p \notin|G| \mid B$ :

$$
B_{1} \in B^{\prime}(G, p) \text { iff }|G|^{\prime} \mid B_{1} \in B^{\prime \prime}(\tilde{G}, p),
$$

and

$$
B_{2} \in B^{\prime \prime}(G, p) \text { iff }|G|^{\prime} \mid B_{2} \in \boldsymbol{B}^{\prime}(\widetilde{G}, p) .
$$

Hence, $B \in \boldsymbol{B}^{\prime \prime}(F)$ iff $|F|^{\prime} \mid B \in \boldsymbol{B}^{\prime}(S(\tilde{G}, \tilde{H}))$

$$
\begin{aligned}
& \text { iff }\left(|F|^{\prime} \mid B\right) \cap|G|=|G|^{\prime} \mid B \in \boldsymbol{B}^{\prime}(\tilde{G}) \\
& \quad \text { and }\left(|F|^{\prime} \mid B\right) \cap|H|=|H|^{\prime} \mid B \in \boldsymbol{B}^{\prime}(\tilde{H}) \\
& \text { iff } B \cap|G|^{\prime} \in \boldsymbol{B}^{\prime \prime}(G) \text { and } B \cap|H|^{\prime} \in \boldsymbol{B}^{\prime \prime}(H) \\
& \text { iff } B \in \boldsymbol{B}^{\prime \prime}(G) \times \boldsymbol{B}^{\prime \prime}(H) \text {. }
\end{aligned}
$$

The case when $B$ is in the family $\boldsymbol{B}^{\prime}(F)$ is proved similarly where, e.g., bases in the family $\boldsymbol{B}^{\prime}(G) \times \boldsymbol{B}^{\prime \prime}(H)$ in $P(G, H)$ come from bases in the family $\boldsymbol{B}^{\prime \prime}(\tilde{G}) \times \boldsymbol{B}^{\prime}(\tilde{H})$ $\times\{p\}$ in $S(\tilde{G}, \tilde{H})$.

If $p$ is a loop of $G$, then, by duality, $\boldsymbol{B}^{\prime \prime}(F)$ is empty, while $\boldsymbol{B}^{\prime}(F)=\boldsymbol{B}^{\prime}(G) \times \boldsymbol{B}^{\prime}(H)$ if $p$ is a loop of $H$, and $\boldsymbol{B}^{\prime}(F)=\boldsymbol{B}^{\prime}(G) \times \boldsymbol{B}^{\prime \prime}(H)$ otherwise.

Corollary 5.3. The contraction $S(G, H) / p$ is equal to the deletion $P(G, H) \mid p$.

Proof. The families $\boldsymbol{B}^{\prime \prime}(S)$ and $\boldsymbol{B}^{\prime}(P)$ are equal when the basepoint $p$ is not an isthmus of $S(G, H)$, and otherwise both pregeometries are the direct sum of the deletions $G \mid p$ and $H \mid p$.

Proposition 5.4. If $(H, p)$ is an isthmus, then $P(G, H)=G$, so the operations of series and parallel connections are both commutative, associative operations with identities.

Proof. Similar to (4.3).

There are a number of propositions for parallel connections analogous to similar propositions in $\$ 4$ for series connections which follow trivially from duality; the facts that $(F / e)^{\sim}=\tilde{F} \mid e,(F \mid e)^{\sim}=\tilde{F} / e,(G \oplus H)^{\sim}=\tilde{G} \oplus \tilde{H}$; and the appropriate propositions in $\S 4$.

Proposition 5.5. If $\|F\| \geqq 2$, then $F=P(G, H)$ is connected iff both $G$ and $H$ are connected.

Proposition 5.6. If $e$ is any point in $|G|^{\prime}$ and $F=P(G, H)$ then contraction and deletion of e commute with the parallel connection: $F / e=P(G / e, H)$ and $F \mid e$ $=P(G \mid e, H)$.

Proposition 5.7. If $F=P(G, H)$ and the basepoint $p$ is not a loop of $G$, then $F \backslash|G|^{\prime}=H$. Hence $(G, p)$ and $(H, p)$ are subgeometries of $P(G, H)$.

Proposition 5.8. If $F=P(G, H)$ then the contraction $F / p$ is separable: $F / p$ 
$=(F / p) \oplus(G / p)$. Further if $F$ is connected and $F / p$ is the direct sum of two pregeometries, $G^{\prime} \oplus H^{\prime}$, then $F$ is isomorphic to the parallel connection $P\left(F \backslash\left|G^{\prime}\right|, F|| H^{\prime} \mid\right)$.

Proposition 5.9. A connected pregeometry $(F, p)$ of more than one point has a unique parallel decomposition into parallel irreducible connected nontrivial pregeometries $\left(G_{1}, p\right), \ldots,\left(G_{n}, p\right)$ such that $F=P\left(G_{1}, \ldots, G_{n}\right)$, where $P$ can be viewed as $a$ symmetric n-ary operator.

Proposition 5.10. If $F=P(G, H)$ and $p$ is not a loop in $G$ or $H$, the circuits of $F$ are given by the following families:

$$
C^{\prime}(F)=\left(C^{\prime \prime}(G) \times C^{\prime \prime}(H)\right) \cup C^{\prime}(G) \cup C^{\prime}(H), \quad C^{\prime \prime}(F)=C^{\prime \prime}(G) \cup C^{\prime \prime}(H) .
$$

Proof. The circuits are the minimal dependent sets (i.e., those which are not contained in any basis). Since the family $\boldsymbol{B}^{\prime \prime}(F)$ equals $\boldsymbol{B}^{\prime \prime}(G) \times \boldsymbol{B}^{\prime \prime}(H)$, a set containing the basepoint $p$ is dependent in $F$ iff its intersection with the subgeometry $(G, p)$ (or $(H, p))$ is dependent in that subgeometry. Hence $C^{\prime \prime}(F)=C^{\prime \prime}(G) \cup C^{\prime \prime}(H)$. Also, since $(G, p)$ and $(H, p)$ are subgeometries of $(F, p), C^{\prime}(F)$ contains the two families $C^{\prime}(G)$ and $C^{\prime}(H)$. If $C=g \cup h$ where $g \in C^{\prime \prime}(G)$ and $h \in C^{\prime \prime}(H)$, then $C$ is dependent by the circuit elimination axiom applied to the circuits $g \cup\{p\}$ and $h \cup\{p\}$. On the other hand, if a circuit $D$ is in $C^{\prime}(F)$, but $g=D \cap|G|$ and $h=D \cap|H|$ are independent and if, e.g., $g \notin C^{\prime \prime}(G)$, then $g \cup\{p\}$ is also independent and $g$ is contained in a set $B \in \boldsymbol{B}^{\prime \prime}(G)$. Thus $D$ is contained in a basis $B^{\prime} \in \boldsymbol{B}^{\prime \prime}(G) \times \boldsymbol{B}^{\prime}(H)-\mathrm{a}$ contradiction. Hence the circuit $D$ is in the family $C^{\prime \prime}(G) \times C^{\prime \prime}(H)$.

Proposition 5.11. The closed sets $K(F)$ of $F=P(G, H)$ are given by the following families:

$$
K^{\prime}(F)=K^{\prime}(G) \times K^{\prime}(H), \quad K^{\prime \prime}(F)=K^{\prime \prime}(G) \times K^{\prime \prime}(H) .
$$

Proof. Assume the basepoint $p$ is not a loop in $G$ or $H$. Then no other sets can be closed since $(G, p)$ and $(H, p)$ are both subgeometries and hence, if the set $K$ is closed in $F$ then the subsets $K \cap|G|$ and $K \cap|H|$ must also be closed, while $p \in K$ iff $p \in K \cap|G|$ and $p \in K \cap|H|$.

None of the above closed sets contains a broken circuit from a circuit in $G$ or $H$. But if $C \in C^{\prime \prime}(G) \times C^{\prime \prime}(H)$ and $D \in K^{\prime}(G) \times K^{\prime}(H)$ and $\|C \mid D\|=1$, then either $\left(C \cap|G|^{\prime}\right) \subseteq\left(D \cap|G|^{\prime}\right)$ or $\left(C \cap|H|^{\prime}\right) \subseteq\left(D \cap|H|^{\prime}\right)$ since the subsets $|G|^{\prime}$ and $|H|^{\prime}$ partition both $C$ and $D$. Assume the former. This means $D \cap|G|^{\prime} \in K^{\prime}(G)$ contains a broken circuit $C^{\prime} \in C^{\prime \prime}(G)$-a contradiction. Consequently, no element of $K^{\prime}(G)$ $\times K^{\prime}(H)$ contains a broken circuit.

Similarly, if $D \in K^{\prime \prime}(G) \times K^{\prime \prime}(H)$, then if $\|C \mid D\|=\|C \backslash(D \cup\{p\})\|=1$, either $\left\|\left(C \cap|G|^{\prime}\right) \mid\left(D \cap|G|^{\prime}\right)\right\|=1$ or $\left\|\left(C \cap|H|^{\prime}\right) \mid\left(D \cap|H|^{\prime}\right)\right\|=1$. Assuming the former we have $\left\|\left(\left(C \cap|G|^{\prime}\right) \cup\{p\}\right) \backslash\left(\left(D \cap|G|^{\prime}\right) \cup\{p\}\right)\right\|=1$. But the former member of the above difference is a circuit in $G$ while the latter is a closed set of $G$. So when $p$ is not a loop, the closed sets are as given above. 
Now assume $p$ is a loop in $G$. Then $p$ is a loop in $F$ and $(F, p)=(H / p) \oplus(G, p)$. Then since $\boldsymbol{K}^{\prime \prime}(H)=\boldsymbol{K}(H / p), \boldsymbol{K}^{\prime \prime}(G)=\boldsymbol{K}(G)$, and $\boldsymbol{K}^{\prime \prime}(F)=\boldsymbol{K}(F): \boldsymbol{K}^{\prime}(F)=\boldsymbol{K}^{\prime}(G) \times \boldsymbol{K}^{\prime}(H)$ vacuously, while $K^{\prime \prime}(F)=K^{\prime \prime}(G) \times K^{\prime \prime}(H)$ by properties of direct sum.

Proposition 5.12. In the category $C$ of pointed pregeometries, direct sums exist and are equal to the parallel connection: $(G, p) \oplus_{C}(H, p)=P((G, p),(H, p))$ $=(P, p)$.

Proof. We must make sure that all goes well in the following commutative diagram:

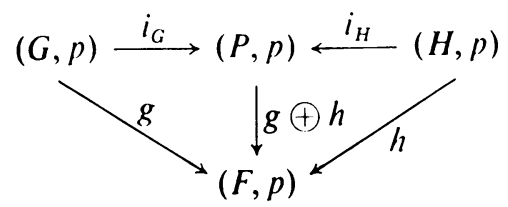

where $(F, p)$ is an arbitrary pregeometry and $g$ and $h$ are arbitrary strong maps. The canonical injection map, $i_{G}$, is strong since if $p$ is a loop in $G$ or not a loop in $H, i_{G}$ is injection of a subgeometry. If $p$ is a loop in $H$ and not in $G$ then $i_{G}$ is contraction by $p$ followed by injection. Similarly for $i_{H}$. For any point $e$ in $(P, p)$ we define $g \oplus h$ by $(g \oplus h)(e)=g(e)$ if $e \in G$ and $h(e)$ if $e \in H$. Then if a closed set $A$ is in $K^{\prime}(F)$, then $g^{-1}(A) \in K^{\prime}(G)$ and $h^{-1}(A) \in K^{\prime}(H)$ since both $g$ and $h$ are strong and preserve $p$. Hence $(g \oplus h)^{-1}(A) \in K^{\prime}(G) \times K^{\prime}(H) \subseteq K(P)$. A similar argument holds for a closed set $A^{\prime} \in K^{\prime \prime}(F) \times\{p\}$. So $g \oplus h$ is strong. The facts that $g$ $=(g \oplus h) \circ\left(i_{G}\right), h=(g \oplus h) \circ\left(i_{H}\right)$, and that $g \oplus h$ is unique follow from point set considerations and noting that the functor to the set category is injective on morphisms.

Note that in the category of pregeometries and strong maps this construction shows that the parallel connection is the pushout from one point.

6. The Tutte-Grothendieck ring. In this section we construct the TutteGrothendieck ring for pointed pregeometries in analogy to the ring for (nonpointed) pregeometries explored in [2].

Definition 6.1. A $\boldsymbol{T}$-invariant $f$ is an invariant defined on the category $\boldsymbol{G}$ of pregeometries taking values in a commutative ring $R$ such that for all pregeometries $G, G_{1}$, and $G_{2}, f(G)=f(G \mid e)+f(G / e)$ when the point $e \in G$ is neither an isthmus nor a loop, and $f\left(G_{1} \oplus G_{2}\right)=\left(f\left(G_{1}\right)\right)\left(f\left(G_{2}\right)\right)$.

Examples of $\boldsymbol{T}$-invariants into the integers include $c(G)$, the complexity or number of bases of $G ; I(G)$, the number of independent sets; $(-1)^{r(G)} \mu(G)$, the absolute value of the Möbius function and $(-1)^{r(G)} \chi(G)$ where $\chi(G)$ is the chromatic polynomial. Others are found in [2].

Definition 6.2. In [2], the Tutte-Grothendieck ring, $T$, is defined as the quotient ring $\boldsymbol{R} / \boldsymbol{I}$ where $\boldsymbol{R}$ is the free commutative ring (without unit) generated by $\boldsymbol{P}$, 
the isomorphism classes of (nonpointed) pregeometries; and $\boldsymbol{I}$ is the ideal generated by ring elements of the form $\left[G_{1}\right]\left[G_{2}\right]-[G]$ and $[H]-[H / e]-[H \mid e]$ for all $\left[G_{1} \oplus G_{2}\right]$ $=[G]$ and for all points $e$ in $H$ where $e$ is neither an isthmus nor a loop.

$\boldsymbol{T}$ has the universal property that there is a 1-1 correspondence of $\boldsymbol{T}$-invariants $f: \boldsymbol{P} \rightarrow R$ and ring homomorphisms $f^{*}: \boldsymbol{T} \rightarrow R$ such that the following diagram commutes:

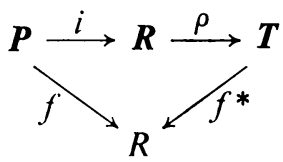

where $i$ injects a pregeometry into $R$ and $\rho$ is the canonical epimorphism. The ring $T$ is isomorphic to $P[z, x]$, the ring of polynomials in two variables over the integers without constant term. For the examples above, if $(\rho \circ i)(G)=f(z, x)$, then $c(G)$ $=f(1,1), I(G)=f(2,1),|\mu(G)|=f(1,0)$, and $(-1)^{r(G)} \chi(G)=f(1-\lambda, 0)$. In addition $\beta(G)=\partial f /\left.\partial z\right|_{x=0, z=0}$.

Definition 6.3. Let $\boldsymbol{G}^{\prime}$ be the category of pointed pregeometries and strong maps $f:\left(F, p_{F}\right) \rightarrow\left(G, p_{G}\right)$ such that not only $f\left(p_{F}\right)=p_{G}$ but $f^{-1}\left(p_{G}\right)=p_{F}$. In this category parallel connection is still direct sum since the canonical injections satisfy the added constraint. There are three distinguished functors from $\boldsymbol{G}^{\prime}$ to $\boldsymbol{G}$, the category of pregeometries and strong maps.

The functor $T: \boldsymbol{G}^{\prime} \rightarrow \boldsymbol{G}$ is the forgetful functor which sends a pointed pregeometry $(F, p)$ to its underlying (nonpointed) pregeometry $F$, and a strong map $f:(G, p) \rightarrow(H, p)$ to its underlying strong map $f: G \rightarrow H$.

The functor $T^{\prime}$ deletes the distinguished element, so $T^{\prime}((F, p))=F \mid p$ and $T^{\prime}(f:(G, p) \rightarrow(H, p))=f^{\prime}:(G \mid p) \rightarrow(H \mid p)$, where $f^{\prime}$ as a set function is the restriction of $f$ to the subset $|G| p \mid$; $f$ is strong since the closed sets of the delion $H \mid p$ are $K^{\prime}(H) \cup K^{\prime \prime}(H)$ and inverse images under $f$ of the former are in $K^{\prime}(G)$ and of the latter in $K^{\prime \prime}(G)$.

$T^{\prime \prime}$ is the functor which contracts by the distinguished element $T^{\prime \prime}((F, p))=F / p$ while $T^{\prime \prime}(f: G \rightarrow H)=f^{\prime \prime}: G / p \rightarrow H / p$, where $f^{\prime \prime}$ as a set function is again the restriction of $f$ to $|G| p \mid$. The morphism $f^{\prime \prime}$ is strong in $\boldsymbol{G}$ since a set $C$ is closed in the contraction $G / p$ iff $C \cup\{p\}$ is closed in $(G, p)$.

Definition 6.4. A $\boldsymbol{T}^{\prime}$-invariant defined on $\boldsymbol{P}^{\prime}$, the set of isomorphism classes of pointed and ordinary pregeometries, and taking values in a commutative ring, is a $\boldsymbol{T}$-invariant on ordinary pregeometries; and for a pointed pregeometry satisfies the following identities: $f((F, p))=f((F \mid e, p))+f((F / e, p))$ for all $(F, p) ; e \in|F|^{\prime}$, $e$ not an isthmus or loop; and $f((F, p) \oplus G)=(f(F, p))(f(G))$ for all pointed pregeometries $(F, p)$ and (nonpointed) $G$ where $(F, p) \oplus G=(F \oplus G, p)$.

Examples of $\boldsymbol{T}^{\prime}$-invariants include $f \circ T$ where $f$ is a $T$-invariant and $T$ is the forgetful functor.

Definition 6.5. Let $\boldsymbol{P}^{\prime}$ be the set of isomorphism classes of pointed and ordinary pregeometries, and $\boldsymbol{R}^{\prime}$ the free comutative ring (without unit) generated by the 
elements of $\boldsymbol{P}^{\prime}$. Let $i: \boldsymbol{P}^{\prime} \rightarrow \boldsymbol{R}^{\prime}$ be the canonical injection and $\boldsymbol{I}^{\prime}$ the ideal of $\boldsymbol{R}^{\prime}$ generated by elements of the forms:

$$
\begin{aligned}
{[(F, p)]-[(F, p) \backslash f]-[(F, p) / f] ; } & {[(F \oplus G, p)]-[(F, p)][G] ; } \\
{[G]-[G \mid e]-[G / e] ; } & \text { and } \quad[G \oplus H]-[G][H]
\end{aligned}
$$

for all pregeometries $G, G^{\prime}$; pointed pregeometries $(F, p)$; and for all points $e \in G$, $f \in|F| p \mid$ where neither $e$ nor $f$ is a loop or isthmus. Let $\boldsymbol{T}^{\prime}$ be the quotient ring $\boldsymbol{R}^{\prime} / \boldsymbol{I}^{\prime}$ and $\rho: \boldsymbol{R}^{\prime} \rightarrow \boldsymbol{T}^{\prime}$ the canonical ring epimorphism $\rho(r)=r+\boldsymbol{I}^{\prime}$. If $t=\rho \circ i$, then $\left(\boldsymbol{T}^{\prime}, t\right)$ is universal in the following sense: for all commutative rings $R$ and for all ring homomorphisms $f^{*}: \boldsymbol{T}^{\prime} \rightarrow R, f=f^{*} \circ t: \boldsymbol{P}^{\prime} \rightarrow R$ is a $\boldsymbol{T}^{\prime}$-invariant. If any other pair $\left(\boldsymbol{T}^{\prime \prime}, t^{\prime}\right)$ has this property, there is a unique ring homomorphism $h: \boldsymbol{T}^{\prime} \rightarrow \boldsymbol{T}^{\prime \prime}$ such that $t^{\prime}=h \circ t$ in the following commutative diagram:

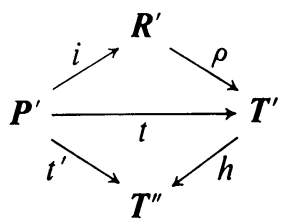

Definition 6.6. Let $Q$ denote the class of polynomials whose variables are pointed and nonpointed pregeometries over the integers (without constant term). For a point $p^{\prime}$, let $D_{p^{\prime}}: Q \rightarrow Q$ be the partial function whose domain is all polynomials, $q$, in which each term contains exactly one factor $F_{j}$; such that $p^{\prime}$ is in the pregeometry $F_{j}$; and for such a polynomial $q=\sum_{j} \prod_{i} G_{i j} F_{j}, D_{p^{\prime}}(q)=\sum_{j} \prod_{i} G_{i j} F_{j}^{\prime}$ where $F_{j}^{\prime}=\left(p^{\prime}\right)\left(F_{j} \backslash p^{\prime}\right)$ if $p^{\prime}$ is a loop or isthmus and $F_{j}^{\prime}=\left(F_{j} \backslash p^{\prime}\right)+\left(F_{j} / p^{\prime}\right)$ otherwise. For any pointed pregeometry $(F, p)$ and ordering $O$ on the point set $|F| p \mid=\left(p_{1}\right.$, $\left.\ldots, p_{n}\right)$, let $D_{o}(F)=\left(D_{p_{n}} \circ D_{p_{n-1}} \circ \ldots \circ D_{p_{1}}\right)(F)$, which is well defined since for all $j, 1 \leqq j \leqq n, D_{p_{j}}$ is defined on the polynomial $q_{j-1}=\left(D_{p_{j-1}} \circ \cdots \circ D_{p_{1}}\right)(F)$. Similarly, we define $D_{o}(G)$ for an ordering of all the points of a nonpointed pregeometry. Two polynomials of the same length, $\sum_{j} \Pi_{i} G_{i j}$ and $\sum_{j} \prod_{i} G_{i j}^{\prime}$ are said to be equivalent if, for some ordering of the terms, $\left[G_{i j}\right]=\left[G_{i j}^{\prime}\right]$ for all $i, j, G_{i j}$ and $G_{i j}^{\prime}$, so $Q / \sim$ is the set of all polynomials of isomorphism classes of pregeometries (and is setisomorphic to $\boldsymbol{R}^{\prime}$ ).

Let $[q]$ denote the equivalence class of the polynomial $q$; and $z, x, z^{\prime}$ and $x^{\prime}$ the equivalence classes of an isthmus, a loop, a pointed isthmus, and a pointed loop respectively.

LEMMA 6.7. (i) $\left[D_{\circ}(F)\right]$ is a polynomial with positive integer coefficients in the variables $z, x, z^{\prime}, x^{\prime}$.

(ii) $\left[D_{O}(F)\right]=\left[D_{O^{\prime}}(F)\right]$ for any orderings $O$ and $O^{\prime}$ of the points of $|F| p \mid$ (or $|F|$ if $F$ is ordinary).

Proof. We will show this if $F$ is a pointed pregeometry.

(i) Inductively, if $\|(F, p)\|=1$, then $p$ is either a loop or an isthmus and hence $\left[D_{O}(F)\right]=x^{\prime}$ or $z^{\prime}$. Assume (i) for all $\|(F, p)\|<n$ and let $D_{o}(F)=$ $\left(D_{p_{n}} \circ D_{p_{n-1}} \circ \cdots \circ D_{p_{2}}\right)\left(D_{p_{1}}(F)\right)$ which equals $\left(D_{p_{n}} \circ \cdots \circ D_{p_{2}}\right)\left(\left(F \mid p_{1}\right)\left(p_{1}\right)\right)$ if $p_{1}$ 
is an isthmus or loop and equals $\left(D_{p_{n}} \circ \cdots \circ D_{p_{2}}\right)\left(\left(F \mid p_{1}\right)+\left(F / p_{1}\right)\right)$ otherwise. But $F \mid p_{1}$ and $F / p_{1}$ both satisfy the induction hypothesis relative to the induced ordering, $O^{\prime}=\left(p_{2}, \ldots, p_{n}\right)$, and by multiplying $\left[D_{O^{\prime}}\left(F \mid p_{1}\right)\right]$ by $\left[p_{1}\right]=x$ or $z$ in the first case or summing $\left[D_{O^{\prime}}(F \mid p)\right]$ and $\left[D_{O^{\prime}}(F / p)\right]$ in the second case, we are done.

(ii) We can effect any reordering by interchanging consecutive points, so we need only show this for $O=\left(p_{1}, \ldots, p_{k}, p_{k+1}, \ldots, p_{n}\right)$ and $O^{\prime}=\left(p_{1}, \ldots, p_{k+1}\right.$, $\left.p_{k}, \ldots, p_{n}\right)$. But by the way we define the operator $D_{o}$, we need only check that on the relevant variables $F_{i j}$ of

$$
\left(D_{p_{k-1}} \circ \cdots \circ D_{p_{1}}\right)(F), \quad\left(D_{p_{k}} \circ D_{p_{k+1}}\right)\left(F_{i j}\right)=\left(D_{p_{k+1}} \circ D_{p_{k}}\right)\left(F_{i j}\right) .
$$

Let $F_{i j}$ be any pointed pregeometry containing the points $p_{k}$ and $p_{k+1}$.

Case I. If $p_{k}$ is neither an isthmus nor a loop of $F_{i j}$, and $p_{k+1}$ is neither an isthmus nor a loop of $F_{i j} \backslash p_{k}$ or $F_{i j} / p_{k}$, then, in $F_{i j}, p_{k}$ and $p_{k+1}$ are not a two-point circuit, neither $p_{k}$ nor $p_{k+1}$ is an isthmus or loop, and there must be a circuit $C_{1}$ containing $p_{k+1}$ that does not contain $p_{k}$. But $p_{k}$ is in some circuit $C_{2}$ and using circuit exchange with $C_{1}$ if necessary, we see that $p_{k}$ must be in a circuit that does not contain $p_{k+1}$. Hence, $p_{k+1}$ is neither an isthmus nor a loop of $F_{i j}$ and $p_{k}$ is neither an isthmus nor a loop of $F_{i j} \backslash p_{k+1}$ or $F_{i j} / p_{k+1}$. By looking at the set of bases we have

$$
\begin{array}{lll}
\left(F_{i j} \backslash p_{k}\right) \backslash p_{k+1}=\left(F_{i j} \backslash p_{k+1}\right) \backslash p_{k} ; & & \left(F_{i j} \mid p_{k}\right) / p_{k+1}=\left(F_{i j} / p_{k+1}\right) \backslash p_{k} \\
\left(F_{i j} / p_{k}\right) / p_{k+1}=\left(F_{i j} / p_{k+1}\right) / p_{k} ; & \text { and } & \left(F_{i j} / p_{k}\right) \backslash p_{k+1}=\left(F_{i j} \backslash p_{k+1}\right) / p_{k}
\end{array}
$$

(e.g., both sides of the last identity define the pregeometry $G$ on the point set $\left|F_{i j}\right|\left(p_{k} \cup p_{k+1}\right) \mid$ with bases, those subsets of $|G|$ which when adjoined with $p_{k}$ form a basis for $F_{i j}$ ).

Case II. If $p_{k}$ is a loop (isthmus) of $F_{i j}$, then it is a loop (isthmus) of $F_{i j} \backslash p_{k+1}$ and $F_{i j} / p_{k+1}$. In any case, $\left(\left(D_{k+1}\left(F_{i j}\right)\right) \backslash p_{k}\right)\left(p_{k}\right)=D_{k+1}\left(\left(F_{i j} \backslash p_{k}\right)\left(p_{k}\right)\right)$.

Case III. If neither $p_{k}$ nor $p_{k+1}$ is a loop or isthmus of $F_{i j}$ but $p_{k+1}$ is a loop of $F_{i j} / p_{k}\left(p_{k+1}\right.$ is an isthmus of $\left.F_{i j} \backslash p_{k}\right)$, then $\left\{p_{k+1}, p_{k}\right\}$ forms a circuit (every circuit containing $p_{k+1}$ contains $\left.p_{k}\right)$. But this means that in $F_{i j}\left(\tilde{F}_{i j}\right), \bar{p}_{k}=\bar{p}_{k+1}$ and there is a strong map automorphism of $F_{i j}$ which interchanges $p_{k}$ and $p_{k+1}$. Clearly, then $\left[D_{O}(F)\right]=\left[D_{O^{\prime}}(F)\right]$.

These are all possible cases so we may denote $\left[D_{O}(F)\right]$ unambiguously by $D(F)$, the (pointed) Tutte polynomial of $(F, p)$.

LEMMA 6.8. If $P^{\prime}=P^{\prime}\left(z, x, z^{\prime}, x^{\prime}\right)$ is the polynomial ring over the integers without constant term and $t^{\prime}: \boldsymbol{P}^{\prime} \rightarrow P^{\prime}$ is the function that sends a pregeometry $F$ to its Tutte polynomial $D(F)$, then for any commutative ring $R$ and homomorphism $h: P^{\prime} \rightarrow R$, $h \circ t^{\prime}$ is a $T^{\prime}$-invariant.

Proof. From properties of $D$, if an ordinary point of $F$ is neither a loop nor isthmus, $D(F \mid e)+D(F / e)=D(F)$ by letting $e=p_{1}$ in $O(F)$. Further, $D(F \oplus G)=$ $D((F)(D(G)))=D(F) D(G)$ if $G$ is a nonpointed pregeometry by letting $O(F \oplus G)=$ $(O(G), O(F))$. Hence, $h \circ t^{\prime}(F)=h(D(F))=h(D(F \mid e)+D(F / e))=h(D(F \mid e))+h(D(F / e))$ 


$$
\begin{aligned}
& =\left(h \circ t^{\prime}\right)(F \mid e)+\left(h \circ t^{\prime}\right)(F / e) \quad \text { and } \quad h \circ t^{\prime}(F \oplus G)=h(D(F \oplus G))=h(D(F) D(G)) \\
& =(h(D(F)))(h(D(G)))=\left(\left(h \circ t^{\prime}\right)(F)\right)\left(\left(h \circ t^{\prime}\right)(G)\right)
\end{aligned}
$$

THEOREM 6.9. The (pointed) Tutte-Grothendieck ring, $\boldsymbol{T}^{\prime}$, is algebraically isomorphic to $P^{\prime}\left(z, x, z^{\prime}, x^{\prime}\right)$, a polynomial ring over the integers in four variables without constant term.

Proof. By the above lemma and (6.5) there exists a homomorphism $h: T^{\prime} \rightarrow P^{\prime}$, such that $h \circ t=t^{\prime}$. By a small notational abuse, denote the cosets in $T^{\prime}$ of an isthmus, loop, pointed isthmus, and pointed loop by $z, x, z^{\prime}$, and $x^{\prime}$ respectively. Then $h(z)=z, h(x)=x, h\left(z^{\prime}\right)=z^{\prime}$, and $h\left(x^{\prime}\right)=x^{\prime}$. Since $P^{\prime}$ is free on these four variables, there is no relation in $T^{\prime}$ among these cosets. If $t^{\prime}(F)=f\left(z, x, z^{\prime}, x^{\prime}\right)$ it is easily seen (by ordering the points of $F$ and observing that the operators $D_{p_{i}}$ correspond to generators of $\left.I^{\prime}\right)$ that $F=f\left(z, x, z^{\prime}, x^{\prime}\right)\left(\bmod I^{\prime}\right)$ when both are viewed as elements of $\boldsymbol{R}^{\prime}$. Hence any generator of $\boldsymbol{R}^{\prime}$ and so any element of $\boldsymbol{R}^{\prime}$ can be expressed as a sum of products of the above four cosets. We have shown that $\boldsymbol{T}^{\prime}$ is the free commutative ring on four generators and hence is isomorphic to $P^{\prime}$, while $t=t^{\prime}$.

Proposition 6.10. We see by (6.9) that $\boldsymbol{T}^{\prime}$ has the additional property that any $\boldsymbol{T}^{\prime}$-invariant $f$ corresponds to a unique homomorphism $f^{*}$ (which is found by evaluating $f^{*}(z)=$ the value of $f$ at an isthmus, etc.) such that $f=f^{*} \circ t$. We also note that $t((F, p))=z^{\prime} f_{1}(z, x)+x^{\prime} f_{2}(z, x)$ since $D(F)$ leaves $p$ as a one-point factor of every term and hence as a pointed isthmus or loop. The isomorphism classes of nonpointed pregeometries $\boldsymbol{P}$ generate an ideal of $\boldsymbol{T}^{\prime}$ isomorphic to $T: T \simeq P(z, x) \simeq P^{\prime}(z, x, 0,0)$ by the obvious isomorphism.

EXAMPLE 6.11. Since the operation of duality commutes with $t, \tilde{t}(F)=t(\tilde{F})$ is a $T^{\prime}$-invariant from $\boldsymbol{P}^{\prime}$ into $\boldsymbol{T}^{\prime}$. Computing the one-point cases, if $t(F)=f\left(z, x, z^{\prime}, x^{\prime}\right)$ then $z(F)=f\left(x, z, x^{\prime}, z^{\prime}\right)$.

LEMMA 6.12. If $\left(C^{n}, p\right)$ is a circuit of $n$ points (with basepoint), then $t\left(\left(C^{n}, p\right)\right)$ $=z^{\prime}\left(z^{n-2}+z^{n-3}+\cdots+z+1\right)+x^{\prime}$.

If $\left(C_{n}, p\right)$ is the rank one pointed pregeometry in which the empty set is closed and $n-1$ other points are in the closure of $p$, then $t\left(\left(C_{n}, p\right)\right)=z^{\prime}+x^{\prime}\left(x^{n-2}+x^{n-3}+\cdots\right.$ $+x+1)$.

Proof. $t\left(\left(C^{1}, p\right)\right)=t(p)=x^{\prime}$; and for all $n>1, t\left(\left(C^{n}, p\right)\right)=t\left(\left(C^{n}, p\right) \mid e\right)+t\left(\left(C^{n}, p\right) / e\right)$ $=z^{\prime} z^{n-2}+t\left(\left(C^{n-1}, p\right)\right)$.

$\left(C_{n}, p\right)$ is isomorphic to the dual of $\left(C^{n}, p\right)$, hence the second half of the lemma follows from (6.11).

For a pointed pregeometry $F$ and its Tutte polynomial $t(F)$, let $\partial F / \partial z^{\prime}$ $=\partial(t(F)) / \partial z^{\prime}=$ the coefficient of $z^{\prime}$ in $t(F)$. Similarly for $\partial F / \partial x^{\prime}$.

THEOREM 6.13. If $(F, p)$ is a pointed pregeometry and $T, T^{\prime}, T^{\prime \prime}$ are the three 
functors of (6.3), then if the Tutte polynomial of $F, t(F)=z^{\prime} f_{1}(z, x)+x^{\prime} f_{2}(z, x)$ $=z^{\prime} \partial F / \partial z^{\prime}+x^{\prime} \partial F / \partial x^{\prime}$, then

$$
(t \circ T)(F)=z f_{1}+x f_{2} .
$$

If $p$ is either an isthmus or a loop,

$$
\left(t \circ T^{\prime}\right)(F)=\left(t \circ T^{\prime \prime}\right)(F)=f_{1}+f_{2} .
$$

Otherwise, if $p$ is neither an isthmus nor a loop,

$$
\left(t \circ T^{\prime}\right)(F)=(z-1) f_{1}+f_{2}, \quad\left(t \circ T^{\prime \prime}\right)(F)=f_{1}+(x-1) f_{2} .
$$

Proof. The functor $T$ treats $F$ like a nonpointed pregeometry and since $(t \circ T)(F)$ is independent of the way the points of $T(F)$ are ordered, we can make $p$ the last point in the ordering, treating it as an ordinary isthmus or loop.

If $p$ is an isthmus in $F$, then $f_{2}=0$, and $z^{\prime}\left(\left(t \circ T^{\prime}\right)(F)\right)=z^{\prime}(t(F \mid p))=t(p \oplus(F \mid p))$ $=t(F)=z^{\prime} f_{1}=z^{\prime}\left(f_{1}+f_{2}\right)$. The cases where $p$ is a loop and for $T^{\prime \prime}$ are proved analogously.

If $p$ is neither an isthmus nor a loop, then

Case I. If $F=\left(C^{n}, p\right) \oplus G$, then $T^{\prime}(F)$ is the direct sum of $G$ and $n-1$ isthmi; hence $t\left(T^{\prime}(F)\right)=z^{n-1}(t(G))=\left((z-1) f_{1}+f_{2}\right)(t(G))$ by $(6.12)$.

Case II. If $F=\left(C_{n}, p\right) \oplus G, T^{\prime}(F)=\left(C_{n-1}\right) \oplus G$ and

$$
\left(t \circ T^{\prime}\right)(F)=\left(z+x^{n-2}+x^{n-3}+\cdots+x\right)(t(G))=\left((z-1) f_{1}+f_{2}\right)(t(G))
$$

by $(6.12)$.

Case III. In all other cases, if any point $e$ is not an isthmus or a loop, and $e$ is not in the closure of $p$ (i.e., $F$ has no two-point circuit $\{e, p\}$ ) and also $e$ is not incident with all circuits containing $p$, then $p$ is neither an isthmus nor a loop of $(F, p) \mid e$ or $(F, p) / e$ and so, $(F \backslash p) \backslash e=(F \mid e) \backslash p$ and $(F \mid p) / e=(F / e) \backslash p$. Hence $\left(t \circ T^{\prime}\right)(F)=$ $\left(t \circ T^{\prime}\right)(F \mid e)+\left(t \circ T^{\prime}\right)(F / e)$. We can continue to decompose by such points, $e$, as long as the above conditions are met, i.e., until we have a sum of indecomposable pointed pregeometries which must be of the form of those handled in Case I and II above. Then by distributivity the theorem is proved.

$T^{\prime \prime}$ can be shown dually or by the observation that if $p$ is neither a loop nor an isthmus, then $(t \circ T)=\left(t \circ T^{\prime}\right)+\left(t \circ T^{\prime \prime}\right)$ and hence $\left(t \circ T^{\prime \prime}\right)(F)=z f_{1}+x f_{2}-\left((z-1) f_{1}+f_{2}\right)$ $=f_{1}+(x-1) f_{2}$.

COROLlaRY 6.14. If $e$ is neither an isthmus nor a loop of the (nonpointed) pregeometry $G$, and if we know the Tutte polynomials of the deletion and contraction of $G$ by e, $t(G \mid e)=d(z, x)$, and $t(G / e)=c(z, x)$; then we can compute the (pointed) Tutte polynomial of $(G, e)$ :

$$
(x+z-x z) t((G, e))=z^{\prime}(c-(x-1) d)+x^{\prime}(d-(z-1) c) .
$$

Proof. The above is the solution to the simultaneous linear equations found in (6.13): $c=f_{1}+(x-1) f_{2}, d=(z-1) f_{1}+f_{2}$. 
THEOREM 6.15. If in either $\mathrm{G}$ or $H, p$ is neither an isthmus nor a loop, the Tutte polynomials of the series and parallel connections are given as follows:

and

$$
t(S(G, H))=z^{\prime}\left[(z-1) \frac{\partial G}{\partial z^{\prime}} \frac{\partial H}{\partial z^{\prime}}+\frac{\partial G}{\partial x^{\prime}} \frac{\partial H}{\partial z^{\prime}}+\frac{\partial G}{\partial z^{\prime}} \frac{\partial H}{\partial x^{\prime}}\right]+x^{\prime}\left[\frac{\partial G}{\partial x^{\prime}} \frac{\partial H}{\partial x^{\prime}}\right]
$$

$$
t(P(G, H))=z^{\prime}\left[\frac{\partial G}{\partial z^{\prime}} \frac{\partial H}{\partial z^{\prime}}\right]+x^{\prime}\left[(x-1) \frac{\partial G}{\partial x^{\prime}} \frac{\partial H}{\partial x^{\prime}}+\frac{\partial G}{\partial z^{\prime}} \frac{\partial H}{\partial x^{\prime}}+\frac{\partial G}{\partial x^{\prime}} \frac{\partial H}{\partial z^{\prime}}\right]
$$

Proof. We will show this for $F=S(G, H)$. The other case is proved analogously or by (6.11). Assume the basepoint $p$ is neither an isthmus nor a loop in $G$. Then, decomposing $F$ and $H$ simultaneously by points in the deletion $H \backslash p$, if $p$ becomes an isthmus in a term of the decomposition of $H$, then $G \backslash p$ will be a direct sum factor in the corresponding term in the decomposition of $F$. If the basepoint $p$ becomes a loop in a term of the decomposition $D(H)$, then $(G, p)$ will be a direct sum factor in the corresponding term of $D(F)$. Hence a term $p \oplus H_{1}$ will correspond to a term $p \oplus H_{1} \oplus(G \backslash p)$ if $p$ is an isthmus; while if $p$ is a loop, a term $p \oplus H_{2}$ will correspond to $H_{2} \oplus(G, p)$. The former terms in the Tutte polynomial are found in $\partial H / \partial z^{\prime}$ while the latter are found in $\partial H / \partial x^{\prime}$. Hence,

$$
\begin{aligned}
t(F) & =\frac{\partial H}{\partial z^{\prime}} z^{\prime}\left[\left(t \circ T^{\prime}\right)(G)\right]+\frac{\partial H}{\partial x^{\prime}}[t(G)] \\
& =\frac{\partial H}{\partial z^{\prime}} z^{\prime}\left[(z-1) \frac{\partial G}{\partial z^{\prime}}+\frac{\partial G}{\partial x^{\prime}}\right]+\frac{\partial H}{\partial x^{\prime}}\left[z^{\prime} \frac{\partial G}{\partial z^{\prime}}+x^{\prime} \frac{\partial G}{\partial x^{\prime}}\right] .
\end{aligned}
$$

THEOREM 6.16. If $P=P(G, H)$ and $S=S(G, H)$, and the basepoint $p$ is neither an isthmus nor a loop in $G$ or $H$, the following invariants can be computed:

(i) The rank function: $r(P)=r(G)+r(H)-1, r(S)=r(G)+r(H)$.

(ii) The Möbius function: $\mu(P)=-\mu(G) \mu(H), \mu(S)=\mu(G) \mu(H \mid p)+\mu(G \backslash p) \mu(H)$.

(iii) The number of independent sets: $I(P)=I(G \backslash p) I(H / p)+I(G / p) I(H \mid p), I(S)$ $=I(G) I(H)-2 I(G / p) I(H / p)$.

(iv) The number of bases: $c(P)=c(G) c(H)-c(G \backslash p) c(H \backslash p), \quad c(S)=c(G) c(H)$ $-c(G / p) c(H / p)$.

(v) The chromatic polynomial: $\chi(P)=\chi(G) \chi(H) /(\lambda-1)$,

$$
\chi(S)=((\lambda-2) /(\lambda-1)) \chi(G) \chi(H)+\chi(G) \chi(H / p)+\chi(G / p) \chi(H) .
$$

(vi) $\beta(P)=\beta(S)=\beta(G) \beta(H)$.

Proof. In the following, we will make continual use of evaluations of the Tutte polynomial in (6.2) as well as the formulas in (6.13) and (6.15). Let $p\left(z, x, z^{\prime}, x^{\prime}\right)$ and $s\left(z, x, z^{\prime}, x^{\prime}\right)$ denote the Tutte polynomials of $(P, p)$ and $(S, p)$ respectively; and let $g_{1}=e\left(\partial G / \partial z^{\prime}\right), g_{2}=e\left(\partial G / \partial x^{\prime}\right), h_{1}=e\left(\partial H / \partial z^{\prime}\right)$, and $h_{2}=e\left(\partial H / \partial x^{\prime}\right)$, where $e$ is the evaluation corresponding to the $\boldsymbol{T}^{\prime}$-invariant under consideration.

(i) $r(P)-1=r(P / p)=r(G / p \oplus H / p)=r(G)-1+r(H)-1$ by $(5.8)$.

(i) $r(S)=r(S \mid p)=f(G|p \oplus H| p)=r(G)+r(H)$ by (4.9). 
(ii) $(-1)^{r(G)+r(H)-1} \mu(P)=(-1)^{r(P)} \mu(P)=p(1,0,1,0)=g_{1} h_{1}=(-1)^{r(G)+r(H)} \mu(G) \mu(H)$.

(ii) $(-1)^{r(S)} \mu(S)=s(1,0,1,0)=g_{1} h_{2}+g_{2} h_{1}$. But $\mu(G)=(-1)^{r(G)} g_{1}$ and $\mu(H \mid p)$ $=(-1)^{r(H \backslash p)}\left((1-1) h_{1}+h_{2}\right)=(-1)^{r(H)} h_{2}$, so $g_{1} h_{2}=(-1)^{r(S)} \mu(G) \mu(H \backslash p)$ and similarly for $g_{2} h_{1}$.

(iii) $I(G \mid p)=(2-1) g_{1}+g_{2}$ and $I(H / p)=h_{1}+(1-1) h_{2}$. Hence $I(G \mid p) I(H / p)+$ $I(G / p) I(H \mid p)=\left(g_{1} h_{1}+g_{2} h_{1}\right)+\left(g_{1} h_{1}+g_{1} h_{2}\right)=2 g_{1} h_{1}+g_{1} h_{2}+g_{2} h_{1}=p(2,1,2,1)=I(P)$.

(iii)' $I(S)=s(2,1,2,1)=2\left(g_{1} h_{1}+g_{1} h_{2}+g_{2} h_{1}\right)+g_{2} h_{2}$. But $I(G) I(H)=\left(2 g_{1}+g_{2}\right)$ $\cdot\left(2 h_{1}+h_{2}\right)=I(S)+2 g_{1} h_{1}=I(S)+2 I(G / p) I(H / p)$.

(iv) We may evaluate at $(1,1,1,1)$ or use (5.2) and (4.4).

(v) $(-1)^{r(P)} \chi(P)=p(1-\lambda, 0,1-\lambda, 0)=(1-\lambda) g_{1} h_{1}$. But $(1-\lambda) g_{1}=(-1)^{r(G)} \chi(G)$ and $\quad(1-\lambda) h_{1}=(-1)^{r(H)} \chi(H)$. Hence, $\quad(\lambda-1) \chi(P)=(-1)^{r(G)+r(H)}(1-\lambda)^{2} g_{1} h_{1}=$ $\chi(G) \chi(H)$.

$$
\begin{aligned}
(-1)^{r(S)} \chi(S) & =s(1-\lambda, 0,1-\lambda, 0) \\
& =(1-\lambda)\left(-\lambda g_{1} h_{1}+g_{1} h_{2}+g_{2} h_{1}\right) \\
& =(\lambda-1)\left((\lambda-2) g_{1} h_{1}+g_{1}\left(h_{1}-h_{2}\right)+h_{1}\left(g_{1}-g_{2}\right)\right)
\end{aligned}
$$

But, as above, $g_{1}=(-1)^{r(G)-1} \chi(G) /(\lambda-1)$, while $(-1)^{r(G)-1} \chi(H / p)=h_{1}+(0-1) h_{2}$. Hence $(\lambda-1)^{2} g_{1} h_{1}=(-1)^{r(S)} \chi(G) \chi(H) ;(\lambda-1) g_{1}\left(h_{1}-h_{2}\right)=(-1)^{r(S)} \chi(G) \chi(H / p)$; and $(\lambda-1) h_{1}\left(g_{1}-g_{2}\right)=(-1)^{r(S)} \chi(H) \chi(G / p)$.

$$
\begin{aligned}
\beta(P) & =\left.\frac{\partial P}{\partial z}((t \circ T)(P))\right|_{x=z=0}=\left.\frac{\partial P}{\partial z}\left(z \frac{\partial P}{\partial z^{\prime}}+x \frac{\partial P}{\partial x^{\prime}}\right)\right|_{x=z=0} \\
& =\left.\frac{\partial P}{\partial z^{\prime}}\right|_{x=z=0}=\left.\frac{\partial G}{\partial z^{\prime}} \frac{\partial H}{\partial z^{\prime}}\right|_{x=z=0}=\beta(G) \beta(H) .
\end{aligned}
$$

(vi)' Since $\beta$ gives the same value for a pregeometry $F$ and its dual, $\tilde{F}$, for all $\|F\|>1, \beta(S)=\beta(\tilde{S})=\beta(P(\tilde{G}, \tilde{H}))=\beta(\tilde{G}) \beta(\tilde{H})=\beta(G) \beta(H)$.

7. Series-parallel networks. We will now define a class of pregeometries which represent the graphs of series-parallel networks. We characterize such networks by a number of equivalent conditions and then investigate various invariants on the class by use of the Tutte-Grothendieck ring.

Definition 7.1. On the set $A$ of isomorphism classes of connected pointed pregeometries with at least two points (hence $p$ is in a circuit) we define the algebra $A=(A, P, S)$ of $A$ and the two commutative semigroup operators $P(\cdot, \cdot)$ and $S(\cdot, \cdot)$ (which are closed by (5.5) and (4.6)).

$\boldsymbol{A}$ is free in the following sense: for no four elements $G_{1}, G_{2}, H_{1}$ and $H_{2}$ of $\boldsymbol{A}$ can the following identity hold: $P\left(G_{1}, H_{1}\right)=S\left(G_{2}, H_{2}\right)$; since if $G_{1}, H_{1}$ and $F$ $=P\left(G_{1}, H_{1}\right)$ are all connected, then the deletion $F \mid p$ is connected, any two points being contained in a common circuit of $C^{\prime}(F)$. Hence by (4.9), $F$ is not a nontrivial series connection. The above remarks along with (4.11) and (5.9) prove the following theorem: 
THEOREM 7.2. An element $F$ of $\boldsymbol{A}$ has a unique series-parallel decomposition into series-parallel irreducible elements. $\boldsymbol{A}$ is also dual closed.

Definition 7.3. Using (7.2) we say $(F, p) \in A$ is essentially series or essentially parallel if the deletion $F \mid p$ is separable or if the contraction $F / p$ is separable respectively. If both minors are connected, $(F, p)$ is then series-parallel irreducible.

Definition 7.4. Denote by $A\left[C_{2}\right]$ the subalgebra of $\boldsymbol{A}$ generated by $C_{2}$, the twopoint circuit. A pointed pregeometry $(F, p)$ is called a series-parallel network if $(F, p) \in A\left[C_{2}\right]$. A (nonpointed) pregeometry, $G$, is termed a series-parallel network if for some point $e \in G$, the pointed pregeometry $(G, e)$ is a series-parallel network.

Proposition 7.5. Any connected minor $(K, p),\|K\| \geqq 2$, of $(F, p) \in A\left[C_{2}\right]$ containing $p$ is also an element of $A\left[C_{2}\right]$.

Proof. We use induction on the number of points, $\|F\|$, in $(F, p)$. The proposition holds trivially for $C_{2}$. Assume that $(F, p)$ is a smallest series-parallel network with a connected minor $(K, p)$ which is not in $A\left[C_{2}\right]$. We may assume $F=S(G, H)$ where $\|G\|<\|F\|$ and $\|H\|<\|F\|$. Then $(K, p)$ is formed by a sequence of contractions and deletions of points in $|F| \mid\{p\}$. But by $(4.7),(K, p)=S\left(K^{\prime}, K^{\prime \prime}\right)$ where $\left(K^{\prime}, p\right)$ and $\left(K^{\prime \prime}, p\right)$ are minors of $(G, p)$ and $(H, p)$ respectively. Also, by (4.6), $\left(K^{\prime}, p\right)$ and $\left(K^{\prime \prime}, p\right)$ are both connected; so by induction, $K^{\prime}$ and $K^{\prime \prime}$ are both in $A\left[C_{2}\right]$, hence so is $K$. The case $F=P(G, H)$ is proved identically.

THEOREM 7.6. A connected pregeometry (without basepoint), $G$, of two or more points is a series-parallel network iff it satisfies one of the following equivalent conditions:

(1) It is a series-parallel network relative to some point.

(2) $\beta(G)=1$.

(3) It is a series-parallel network relative to any point.

(4) For any connected minor $K$ of $G$ such that $\|K\| \geqq 2 ; K$ or $\tilde{K}$ is not a geometry (i.e., the individual points of $K$ or $\widetilde{K}$ are not all closed).

(5) No minor $K$ of $G$ is isomorphic to $L_{4}$ (the four-point line) or $P_{4}$ (the geometry of the partitions of a four-element set).

(6) For any connected minor $K$ of $G(\|K\|>2)$, and any point $e \in K ; K \mid e$ or $K / e$ is separable.

Note. (2) shows that $A\left[C_{2}\right]$ can be completely characterized in the TutteGrothendieck ring.

(4) implies that $A\left[C_{2}\right]$ can be constructed inductively from $C_{2}$ by adding points in parallel (i.e., replacing points with two-point circuits), and dualizing.

(5) implies that $A\left[C_{2}\right]$ is contained in the class of planar graphical pregeometries, since $P_{4}$ is the canonical geometry associated with the pregeometries $P_{5} / e, F \mid e$, and $\left(P_{3,3} / f_{1}\right) / f_{2} ;$ where $P_{5}$ is the geometry of partitions of a five-element set; $F$ is the Fano projective plane of seven points, and $P_{3,3}$ is the geometry of contractions of the Kuratowski complete bipartite graph where $f_{1}$ and $f_{2}$ are any two edges not incident 
with the same vertex. $P_{4}$ is also self-dual; and hence $P_{4}$ is a minor of $P_{5}, \tilde{P}_{5}, P_{3,3}, \tilde{P}_{3,3}$, $F$, and $\tilde{F}$; and since $A\left[C_{2}\right]$ can contain none of the above as minors as well as no fourpoint line; the Tutte representation theorem [16] gives the implication. Also it is a trivial consequence of (5) that connected nontrivial minors of series-parallel networks are series-parallel networks.

Proof of Theorem. (1) $\Rightarrow(2)$. In (6.16) we showed that for all $G, H \in A\left[C_{2}\right]$, $\beta(S(G, H))=\beta(P(G, H))=\beta(G) \beta(H)$. But $\beta\left(C_{2}\right)=1$.

(2) $\Rightarrow$ (3). If $\|G\|=2$ and $G$ is connected, then $G=C_{2}$ and $C_{2}$ is series-parallel relative to both its points. Assume the theorem for all pregeometries $\left\|G_{i}\right\|<n$ and let $\|G\|=n$ and $\beta(G)=1$. Then for any point $e$ in $G, e$ is neither a loop nor an isthmus and $\beta(G \mid e)$ or $\beta(G / e)=0$. Assume the former. Then, since the deletion $G \mid e$ is separable, $(G, e)=S\left(\left(G_{1}, e\right),\left(G_{2}, e\right)\right)$ by $(4.10)$ where $n>\left\|G_{i}\right\| \geqq 2$, for $i=1,2$, and both are connected. But in $(6.16(\mathrm{vi}))$ we showed that under these conditions $\beta(G)$ $=\beta\left(G_{1}\right) \beta\left(G_{2}\right)$ and since this could only happen if $\beta\left(G_{1}\right)=\beta\left(G_{2}\right)=1$ we are done by induction. The same holds if $\beta(G / e)=0$.

(3) $\Rightarrow(4) . C_{2}=\tilde{C}_{2}$ is not a geometry. We will prove the stronger result that for any larger connected minor, $K$, either $K$ or its dual $\widetilde{K}$ contains a two-point circuit disjoint from any given point $e \in K$. Assume $K$ is a connected minor of $G,\|K\| \geqq 3$ and $e \in K$. Then by hypothesis, $(G, e) \in A\left[C_{2}\right]$ and by (7.5), $(K, e) \in A\left[C_{2}\right]$. If $\|K\|=3, K$ is equal to $S\left(C_{2}, C_{2}\right)$ or $P\left(C_{2}, C_{2}\right)=\tilde{S}\left(C_{2}, C_{2}\right)$. In any case $K$ or $\tilde{K}$ equals $P\left(C_{2}, C_{2}\right)$ in which the two ordinary points form a circuit. Assume the conclusion for all $3 \leqq\left\|K_{i}\right\|<n$ and let $\|K\|=n$. If $K=S(G, H)$, we may assume $\|G\| \geqq 3$. By induction, one of the families $C^{\prime}(G)$ or $C^{\prime}(\widetilde{G})$ contains a two-point circuit. But $\tilde{K}=P(\tilde{G}, \tilde{H})$ and both families $C^{\prime}(S)$ and $C^{\prime}(P)$ preserve two-point circuits. Similarly for $K=P(G, H)$.

(4) $\Rightarrow$ (5). $P_{4}=\tilde{P}_{4}$ and $L_{4}=\tilde{L}_{4}$ are both geometries.

$(5) \Rightarrow(6)$. By the above Note we may assume that $G$ can be represented by a (planar) graph and our proof will be graph theoretic. So assume $\|K\| \geqq 3 ; K$ contains the edge $e$; and that $K, K \backslash e$, and $K / e$ are all connected (i.e., two-connected in graph theory). Then $e$ is in a $K$-circuit, $C$, which must have at least two other edges, since $K / e$ has no loops. Let the two vertices incident with $e$ be denoted $v_{1}$ and $v_{2}$ respectively. Denote the edge of $C \mid e$ incident with $v_{1}$ as $e_{1}$ and the edge ircident with $v_{2}$ as $e_{2}$; the edges $e_{1}$ and $e_{2}$ must be in a circuit $D \subseteq K \mid e$. Contraction of $e$ in $K$ identifies $v_{1}$ and $v_{2}$ and so contracts $D$ to two circuits $D_{1}$ and $D_{2}$ both incident with $\overline{v_{1} v_{2}}$ and neither is a loop. Since $K / e$ is connected there must be a path $P$ from a vertex in $D_{1} \mid \overline{v_{1} v_{2}}$ to a vertex in $D_{2} \mid \overline{v_{1} v_{2}}$ which does not contain any other vertex in $D$. But then the subgraph $D \cup P \cup\{e\}$ is topologically homeomorphic to $G_{4}$, the complete graph on four vertices. Since $P_{4}$ is the geometry of contractions of $G_{4}, K$ must contain $P_{4}$ as a minor.

(6) $\Rightarrow$ (1). If $\|G\|=2, G=C_{2}$, the two-point circuit. Otherwise for a point $e \in G$, $G \mid e$ or $G / e$ is separable and so $(G, e)$ is the series or parallel connection of two of its 
minors by (4.10) or (5.8). These minors are connected and hence by induction we may continue decomposing till we reach an element which is series-parallel irreducible which must have cardinality less than 3 and hence must be $C_{2}$.

LEMMA 7.7. If $(F, p)$ is a pointed series-parallel network, then the contraction $F / p$ is a direct sum of loops and series-parallel networks. Further, F/p has all even circuits (and hence no loops) iff all circuits in the family $C^{\prime}(F)$ are even and all circuits in the family $C^{\prime \prime}(F) \times\{p\}$ are odd.

Proof. By induction, $C_{2} / p$ is a loop which is an odd circuit while $C_{2}$ is an even circuit containing the basepoint $p$. If the $F$ of our induction step is the parallel connection $P(G, H)$, then by (5.8), the contraction $F / p$ is isomorphic to the direct sum of the two contractions $G / p$ and $H / p$ both of which satisfy the induction hypothesis, while $F / p$ has even circuits iff both $G / p$ and $H / p$ do iff (by induction) sets in the family $C^{\prime}(G) \cup C^{\prime}(H)$ and sets in the family $C^{\prime \prime}(G) \cup C^{\prime \prime}(H)$ are all even. But then sets in $C^{\prime \prime}(G) \times C^{\prime \prime}(H)$ are also all even; hence sets in $C^{\prime}(F)$ are even, and those in $C^{\prime \prime}(F) \times\{p\}$ are odd.

If on the other hand $F=S(G, H)$, then by (5.3), $F / p=P(G, H) \backslash p$. The latter is a connected minor of a series-parallel network and hence is series-parallel by (7.6). Also the family of circuits, $C(P(G, H) \mid p)=C^{\prime}(P(G, H))=C^{\prime}(G) \cup C^{\prime}(H) \cup\left(C^{\prime \prime}(G)\right.$ $\left.\times C^{\prime \prime}(H)\right)$. But these are exactly $C^{\prime}(F) \cup C^{\prime \prime}(F)$. Hence they are all even iff all circuits in $C^{\prime}(F)$ are even while all circuits in $C^{\prime \prime}(F) \times\{p\}$ are odd.

Definition 7.8. A coloring of a graph (and hence a series-parallel network) is a function from the vertex set of the graph into a set such that adjacent vertices are assigned distinct elements. A graph is $n$-colorable if there exists a coloring into a set with $n$ elements.

THEOREM 7.9. Three colors are sufficient to color the vertices of a series-parallel network. The network is two-colorable iff all its circuits are even.

Proof. Let $\chi_{n}(F)$ denote the evaluation of $\chi(F)$ at $\lambda=n>0$. Then, since $F$ is connected, $n \chi_{n}(F)$ is the number of ways of $n$-coloring $F$. (This is proved in [2] and [12].) Hence, we must show that $\chi_{3}(F)$ is positive for all series-parallel networks $F$, and $\chi_{2}(F)$ is positive iff every circuit in $F$ is even. We use induction and (6.16(v)). $\chi\left(C_{2}\right)=\lambda-1$ and hence $\chi_{3}\left(C_{2}\right)$ and $\chi_{2}\left(C_{2}\right)$ are both positive and $C_{2}$ is an even circuit. If the $F$ of our induction step is $P(G, H)$, then $\chi_{3}(F)=\chi_{3}(G) \chi_{3}(H) / 2$ which by induction is positive since the evaluated polynomials $\chi_{3}(G)$ and $\chi_{3}(H)$ both are. $\chi_{2}(F)$ $=\chi_{2}(G) \chi_{2}(H)$, hence $\chi_{2}(F)$ is positive iff $\chi_{2}(G)$ and $\chi_{2}(H)$ both are iff (by induction) all sets in the families $C^{\prime}(G)$ and $C^{\prime}(H)$ are even while all sets in the families $C^{\prime \prime}(G)$ and $C^{\prime \prime}(H)$ are odd (and hence sets in the family $C^{\prime \prime}(G) \times C^{\prime \prime}(H)$ are even) iff all circuits of $F, C(F)$, are even.

If, on the other hand, $F=S(G, H)$, then using only the first term of the expression for $\chi(S), \chi_{3}(F) \geqq \chi_{3}(G) \chi_{3}(H) / 2$ which is positive by induction. $\chi_{2}(F)$ $=\chi_{2}(G) \chi_{2}(H / p)+\chi_{2}(G / p) \chi_{2}(H)$ will be 0 unless one of the terms is positive. But $F$ 
has even circuits iff the sets in the families $C^{\prime}(G)$ and $C^{\prime}(H)$ are even while sets in $C^{\prime \prime}(G) \times C^{\prime \prime}(H)$ are odd. All sets in the family $C^{\prime \prime}(G) \times C^{\prime \prime}(H)$ are odd iff all sets in $C^{\prime \prime}(G)$ are odd and all sets in $C^{\prime \prime}(H)$ are even or all sets in $C^{\prime \prime}(G)$ are even while those in $C^{\prime \prime}(H)$ are odd. By (7.7), the former case holds iff $G$ and $H / p$ both have even circuits and the latter iff $H$ and $G / p$ have all even circuits. The former case holds iff $G$ and each direct sum factor of $H / p$ have even circuits. But each factor of $H / p$ is a series-parallel network by (7.7), hence by induction and since $\chi\left(F_{1} \oplus F_{2}\right)$ $=\chi\left(F_{1}\right) \chi\left(F_{2}\right), G$ and $H / p$ have all even circuits iff all factors of $\chi_{2}(G) \chi_{2}(H / p)$ are positive. Analogously, $H$ and $G / p$ have all even circuits iff $\chi_{2}(H) \chi_{2}(G / p)$ is positive.

\section{BIBLIOGRAPHY}

1. G. D. Birkhoff, A determinant formula for the number of ways of coloring a map, Ann. of Math. (2) 14 (1913), 42-46.

2. T. Brylawski, Thesis, Dartmouth College, Hanover, N. H.

3. H. Crapo, A higher invariant for matroids, J. Combinatorial Theory 2 (1967), 406-417. MR 35 \#6579.

4. —, Möbius inversion in lattices, Arch. Math. 19 (1968), 595-607.

5. - The Tutte polynomial, Aequationes Math. 3 (1970).

6. - The Möbius function of a lattice, J. Combinatorial Theory 1 (1966), 126-131. MR 33 \#1240.

7. H. Crapo and G.-C. Rota, Combinatorial geometries, preliminary ed., M.I.T. Press, Cambridge, Mass., 1970.

8. G. Dirac, A property of 4-chromatic graphs and some remarks on critical graphs, J. London Math. Soc. 27 (1952), 85-92. MR 13, 572.

9. R. Duffin, Topology of series-parallel networks, J. Math. Anal. Appl. 10 (1965), 303-318. MR 31 \#85.

10. P. A. MacMahon, The combination of resistances, Electrician 28 (1892), 601-602.

11. J. Riordan and C. E. Shannon, The number of two-terminal series-parallel networks, J. Math. Phys. Mass. Inst. Tech. 21 (1942), 83-93. MR 4, 151.

12. G.-C. Rota, On the foundations of combinatorial theory. I. Theory of Möbius functions, Z. Wahrscheinlichkeitstheorie und Verw. Gebiete 2 (1964), 340-368. MR 30 \#4688.

13. - Combinatorial analysis as a theory, Hedrick Lectures, Math. Assoc. of Amer., Summer Meeting, Toronto, 1967.

14. C. E. Shannon, A symbolic analysis of relay switching circuits, Trans. Amer. Inst. Elec. Engrs. 57 (1938), 713-723.

15. W. T. Tutte, $A$ ring in graph theory, Proc. Cambridge Philos. Soc. 43 (1947), 26-40. MR 8, 284.

16. - Lectures on matroids, J. Res. Nat. Bur. Standards Sect. B 69B (1965), 1-47. MR 31 \#4023.

17. H. Whitney, The coloring of graphs, Ann. of Math. (2) 33 (1932), 688-718.

18. —_, A logical expansion in mathematics, Bull. Amer. Math. Soc. 38 (1932), 572-579.

19. - On the abstract properties of linear dependence, Amer. J. Math. 57 (1935), 509533.

Dartmouth College,

HaNOVER, New Hampshire 03755 